\title{
Prognostic importance of pathologic fracture and surgical implications in osteosarcoma: a retrospective clinical study
}

\author{
Sam Hajialilo Sami', Khodamorad Jamshidi', Farshad Abolghasemzadeh Ahangar ${ }^{r *}$
}

Received 1 Dec, 2016, accepted for publication 4 Feb, 2017

\begin{abstract}
Background \& Aims: Osteosarcoma is one of the most prevalent malignant bone tumors . This study is designed to assess the prognostic effect of pathologic fracture and treatment options on survival of patients with osteosarcoma.

Materials \& Methods: In this study, prognosis of 106 patients with limb osteosarcoma, which were treated at three hospitals in Tehran from April to August 2012 was evaluated. Patients were divided in two groups according to the presence of pathologic fracture at the time of diagnosis. Survival, local recurrence and distant metastasis were compared between groups. For patients with pathologic fracture, survival rate was compared according to surgical options (amputation or limb salvage). Chi-square and Independent sample T-test were used to compare means of the groups. Recurrence free and metastasis free survivals and final survival of the patients were calculated using the Kaplan-Meier method. To determine the predictive factors of survival, Cox-Regression analysis and estimating hazard ratio (HR) with $95 \%$ confidence interval were used.

Results: Among 106 patients, 57 cases had not and 49 others had a pathologic fracture at the time of diagnosis. There were no statistically difference between two groups in Survival $(\mathrm{P}$-value $=0.36)$, local recurrence $(\mathrm{P}$-value $=0.91)$ and distant metastasis $(\mathrm{P}$ value $=0.73)$. There was no statistically difference in survival according to surgical options in patients with pathologic fracture $(\mathrm{P}-$ value $=0.85)$.
\end{abstract}

Conclusion: pathologic fracture in limb osteosarcoma is not a poor prognostic factor. In patients with pathologic fracture limb salvage procedures can be performed without adverse effect on survival rate.

KeyWords: osteosarcoma, pathologic fracture, limb salvage, amputation, prognosis

Address: Tehran, Baharestan, Shafa Yahyaeian hospital

Tel: +989124454805

Email: farshada1358@gmail.com

\section{Introduction}

Osteosarcoma is the most common bone tumors in almost all decades of life. In fact, after multiple myeloma tumor, it is the second most common primary bone tumors in adults. The incidence of osteosarcoma is around 1 to 3 million per year. Although this disease can occur at any age, but usually it occurs in the second decade of life with high-grade species. Except periosteal that is more common in women, other types of osteosarcoma

\footnotetext{
${ }^{1}$ Associated professor in orthopaedics tumor surgery, Iran university of medical sciences

${ }^{2}$ Professor in orthopaedics tumor surgery, Iran university of medical sciences

${ }^{3}$ Fellowship in orthopaedics tumor surgery, Shafa Yahyaeian bone and joint research center, Iran university of medical sciences(Corresponding Author)
} 
are more common in the men. The most common sites of osteosarcoma occurrence of distal femur are proximal tibia. The Disease presents as a painless mass with progressive growth and intensifying pain at night. Osteosarcoma diagnosis is based on clinical and paraclinical tools such as X-ray, MRI, bone nuclear scan, CT scan and laboratory tests can better assess tumor and its relation to the surrounding elements and help to know if cancer cells spread to another part of the body.

Revolution has been made in the treatment of osteosarcoma after the emergence of new methods of chemotherapy. Currently 65 to 70 percent of people with a high degree of this disease can be associated with long life. Several factors were reported being effective in its prognosis. The spread of disease and tumor grade are important prognostic factors. The main goal of complete elimination of tumor in surgical treatment of patients requires amputation. Chemotherapy is necessary before and after surgery to destroy small metastases that may exist at the onset of the disease (1).

The prevalence of pathological fractures of organs and before treatment in case of osteosarcoma has been reported between 5 and $10 \%(5,4$ and 1$)$. Pathological fracture has been reported in the past as a debilitating factor for prognosis in organs osteosarcoma (7-2). This hypothesis led the amputation as a primary modality for the safety of the patient (4-2). Since the limb amputation, especially in proximal areas follows severe restrictions on patient's functionality, surgery with a limb salvage tendency led physicians to reevaluate pathologic fracture as a prognostic factor. Some recent studies have shown that with limb salvage surgery is possible without affecting disease prognosis and its recurrence rate (10-8). In this study, the treatment of patients with extremity osteosarcoma was reported. Some of them were pathological fracture and the impact of treatment on the prognosis of patients have examined. Also, the incidences of pathological fracture as well as the results limb amputation in patients with pathological fracture compared with limb salvage procedures were evaluated.

\section{Materials and Methods}

In this retrospective study, the treatment of patients with organs osteosarcoma hospitalized in Shafa, Milad and Pars hospitals in Tehran from April to August 2012 were studied. According to the recorded information, 193 patients with osteosarcoma were admitted. Of 193 patients 32 cases did not complete follow-up, thus they were excluded from the study. Also 47 patients were excluded as they initially were treated at other centers and referred after the recurrence of disease. Regional or distant metastasis in 8 patients have been seen in previous studies, thus they were excluded. Finally 106 patients were enrolled in this study.

Of 106 patients, 49 patients at the onset of the disease and before the treatment had fractures at the tumor site while the remaining 57 patients hadn't any fractures. Patients were divided into two groups, namely "without fracture" and "fracture". In group "without fracture" 35 men and 22 women and in the "fracture" group there were 29 males and 20 females. The two groups were almost identical in terms of gender $(\mathrm{P}=0.8)$.

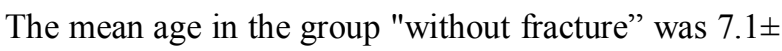
18.3 years (from 6 to 46 years) and in the "fracture" group it was $5.9 \pm 18$ years (from 8 to 32 years). In terms of age, there was no significant difference between two groups $(\mathrm{P}=0.94)$.

The most common sites of disease were distal femur, proximal tibia and proximal humerus. In group "without fracture" in 53 patients (93\%) and in the 
group "fracture" in 44 patients (90\%) the tumor sites were distal femur, proximal tibia and proximal humerus which were similar for both groups $(\mathrm{P}=0.96)$. Information about patients and the comparison between groups are shown in Table 1 .

Core Needle and Open Surgical Biopsy for diagnosis for clinical diagnosis and the pathological assessment for all patients were made. Patients were treated with chemotherapy before surgery. Surgery amputation or limb salvage was carried out. The patients underwent chemotherapy again. After limb salvage surgery, according to patient age, tumor resection site was restructured with allograft or prosthetic. All procedures were performed by one of the two orthopedic surgeons who had experience in the field of tumor surgery. According to the pathology report, in all cases, reasonable margin of healthy tissue around the tumor was removed.

The patients followed-up every 3 months during the first three years and every 6 months over the second three years. At each follow-up examination, lateral chest radiographs and lateral radiographs from the affected limb were performed for any recurrence or metastasis to the lung and in the case of identifying suspicious lesions in the radiographs, CT scan or MRI were performed for further investigation.

In the group "without fracture" 14\% (24.5) of patients amputated and for 43 patients (75.4\%) limb salvage surgeries were performed. In the group "fracture" 15 patients (30.6\%) amputated and in 34 patients (69.4\%) Limb salvage surgeries were performed. The two groups were comparable with regard to the type of performed surgery $(\mathrm{P}=0.22)$.

Patients were followed for 36 months and in case of local recurrence or metastasis, the result of treatment was recorded as failure. Death due to illness or complications was determined as end point of follow-up. The mean follow-up in the group of "without fracture" was $24 \pm 56$ months and in the group of "fracture" it was $28 \pm 57.8$ months that was not statistically significant difference $(\mathrm{P}=0.4)$.

SPSS statistical software (version 0.18) was used to analyze data. Frequency, Mean and Standard deviation were determined. Variables were compared using the Chi-square test. Independent sample T-test was used to compare the two groups. The survival and disease-free period from the time of diagnosis to the time of local recurrence or distant metastasis or death was calculated and evaluated using Kaplan-Meier method. To determine prognostic factors, Cox-regression was used to estimate the hazard ratio with $\% 95$ of confidence interval. Only medical records information was used in this study.

\section{Results}

At the ends of follow-up 58 patients $(54.7 \%)$ were alive. A patient was died due to the heart damage caused by chemotherapy. 47 patients were died because of local recurrence or distant metastasis. In the group "without fracture" 33\% (57.9S) of patients and in the "fracture" group 25 patients (51\%) were alive at the end of follow-up. From this point, no significant difference was between the two groups $(\mathrm{P}=0.36)$.

94 patients of the 106 patients for at least 4 years (48 months) were followed-up. 52 patients out of 94 were from "without fractures" group and 42 patients were from "fractures" group. The 4-year survival rate of patients was 54.3\% (51 live from 94 patients). The survival rates for the two groups, "without fracture" and "fracture" were 59.6\% and $47.6 \%$, respectively. The 4-year survival rate was not significantly different between the two groups. $(\mathrm{P}=0.35)$.

The local recurrence and distant metastasis in these patients were studied. In 38 patients $(35.8 \%)$ one or more local recurrence occurred. The recurrence rate 
for the groups "without fracture" and "fracture" were $35.1 \%$ and $36.7 \%$ which were similar for both groups $(\mathrm{P}=-.91)$. Finally, in $52(49.1 \%)$ patients with distant metastases were observed as follows: for groups, "without fracture" it was $47.4 \%$ and for "fracture" group that was 51\%. This difference wasn't also statistically significant between the two groups $(\mathrm{P}=0.73)$. The results are shown in Table 2 .
In the group "fracture" based on the type of surgery, there was not significant difference in survival between the two groups (amputation or limb salvage) $(\mathrm{P}=0.85)$. Of 15 amputated patients, 8 patients $(53.3 \%)$ and from 34 patients with limb salvage surgery 17 patients $(50 \%)$ were alive at the end of follow-up, see Table 3.

Table 1. All patients in both groups with and without pathological fracture

\begin{tabular}{|c|c|c|c|}
\hline Variables & Without Fracture & With fracture & P-value \\
\hline Frequency & 57 & 49 & - \\
\hline \multirow[t]{2}{*}{ Gender } & Men=35 & Men $=29$ & 0.8 \\
\hline & Woman $=22$ & Woman $=20$ & \\
\hline Age (years) & $18.3 \pm 7.1$ & $18 \pm 5.9$ & 0.94 \\
\hline Location of the tumor (in the common areas $\bullet$ ) & $93 \%$ & $90 \%$ & 0.96 \\
\hline \multirow[t]{2}{*}{ The type of surgery } & Amputation $=14$ & Amputation $=15$ & 0.22 \\
\hline & Limb salvage $=43$ & Limb salvage $=34$ & \\
\hline Follow-up (months( & $24 \pm 56$ & $57.8 \pm 28$ & 0.4 \\
\hline
\end{tabular}

-distal femur, proximal tibia, proximal Humerus

Table 2. Comparison of survival, local recurrence and metastasis between the groups with and without pathological fracture

\begin{tabular}{llll}
\hline Variables & Without fracture & With fracture & P-value \\
\hline Total survival rate & $57.9 \%$ & $51 \%$ & 0.36 \\
Survival rate of 4-year & $59.6 \%$ & $47.6 \%$ & 0.35 \\
Local Recurrence & $35.1 \%$ & $36.7 \%$ & 0.91 \\
Distant metastases & $37 \%$ & $32 \%$ & 9.73 \\
\hline
\end{tabular}

Table 3. The survival rate in patients with pathological fractures based on the type of operation

\begin{tabular}{llll} 
& Amputation & Limb salvage & P-value \\
\hline Total survival rate & $53.3 \%$ & $50 \%$ & 0.85 \\
\hline
\end{tabular}

\section{Discussion and Conclusion}

Based on the results, there was not a significant difference between the two groups, "without fracture" and "fracture" in terms of age, sex, tumor location, surgery type and length of follow up.
Thus, comparison of consequences related to this disease and its outcomes between the two groups shows that the impact of pathological fractures and the type of treatment used in the prognosis of these patients. Our results revealed that pathological 
fracture in osteosarcoma of the extremity increases mortality, local recurrence and distant metastasis, but this impact was not a statistically significant local recurrence and distant metastasis cases. So, it can't be considered pathologic fractures be as a strong debilitating factor in patients with organs osteosarcoma. These results are similar to the study of Griffin et al (11). However, similar studies in this area show different results.

Some studies approved the debilitating effect of pathologic fracture as a prognostic factor of organs osteosarcoma and proposed amputation should be performed primarily as the only chance to save the lives of such patients (7).

Survey within the group of patients with pathological fractures that were present in our study showed that the prognosis of these patients was not affected by the type of surgery. Therefore, it can be concluded that limb salvage surgery can be performed by making sure that patients' prognosis will not be changed. This conclusion is similar to the study of Bacci and colleagues (12).

Scully and colleagues (7) in their paper proposed not using limb salvage surgery in patients with pathological fracture, since it increases the likelihood of tumor cell diffusion and chances of local recurrence and distant metastases and ultimately the reduction in survival rate. But this idea was not confirmed based on our results.

The limitations of this study include patient loss during the follow-up. Since patients did not refer for consultation and the reports were incomplete. It is likely that patients with recurrence or metastasis did not refer or died and if they were referred and their medical records would be complete our results would be better.

Finally, it is suggested that limb assessment and decision on how to treat patients should be limited in each case with osteosarcoma and the decisions should not be influenced by the presence or absence of fracture.

It is recommended that the type of operations (limb salvage or amputation) be chosen considering tumor size and its proximity to nerves and blood vessels.

The limb salvage surgery is the best choice if surgeons prefer to remain vital organs with healthy margins and sufficient removal of the tumor. The main goal of surgery for these patients definitely is complete removal of the tumor with an adequate margin of normal surrounding, otherwise limb amputation should be considered. Because limb salvage surgery, at the expense of incomplete resection of the tumor, increases the chance of local recurrence, metastasis and ultimately patient death. However, randomized prospective clinical studies are needed to confirm such a conclusion.

In this study, we tried to remove factors such as age, sex, tumor location, type of surgery and follow-up that could interfere with the study results and two groups of this study were homogeneous in terms of the above factors.

The presence of pathological fracture in osteosarcoma treatment on the basis of this study is not debilitating prognosis. In these patients in case of fractures, surgery procedures with limb salvage can be performed without having a negative impact on survival rates.

Ethics: All patient information was retrieved from the medical record archive confidentially and patients' identification data were not registered and reported anywhere.

\section{References}

1. Canale ST, Beaty JH. Campbell's Operative Orthopaedics. Elsevier Health Sciences; 2012. volume 1, chapter 22, P.901-7. 
2. Abudu A, Sferopoulos NK, Tillman RM, Carter SR, Grimer

RJ. The surgical treatment and outcome of pathological fractures in localised osteosarcoma. J Bone Joint Surg Br 1996;78(5): 694-8.

3. Coley BL, Pool JL. Factors influencing the prognosis in osteogenic sarcoma. Ann Surg 1940;112(6): 1114-28.

4. Jaffe N, Spears R, Eftekhari F, Robertson R, Cangir A, Takaue Y, et al. Pathologic fracture in osteosarcoma. Impact of chemotherapy on primary tumor and survival. Cancer 1987;59(4): 701-9.

5. Mulder Jo, Schutte HE, Kroon HM, Taconis WK. Radiologic atlas of bone tumors- Amsterdam: Elsevier science; 1993. Intraosseous osteosarcoma: conventional type; P51-5.

6. Scully SP, Temple HT, O’Keefe RJ, Mankin HJ, Gebhardt M. The surgical treatment of patients with osteosarcoma who sustain a pathologic fracture. Clin Orthop Relat Res 1996;(324): 227-32.

7. Scully SP, Ghert MA, Zurakowski D, Thompson RC, Gebhardt MC. Pathologic fracture in osteosarcoma: prognostic importance and treatment implications. J Bone Joint Surg Am 2002;84-A(1): 49-57.

8. Canale ST, Beaty JH. Campbell's Operative Orthopaedics. 12th ed. Elsevier Health Sciences; 2012. volume 1, chapter $19,785-7$.

9. Simon MA. Limb salvage for osteosarcoma. J Bone Joint Surg Am 1988;70(2): 307-10.

10. Simon MA, Aschliman MA, Thomas N, Mankin HJ. Limbsalvage treatment versus amputation for osteosarcoma of the distal end of the femur. J Bone Joint Surg Am 1986;68(9): 1331-7.

11. Griffin Am, Mclaughlin C, Ferguson PC, Bell RS, Wunder JS. Outcome following presentation with a pathologic fracture in osteosarcoma.J Bone Joint Surg Br 2008; 90: $87-8$.

12. Bacci G, Ferrari S, Longhi A, Donati D, Manfrini M. Nonmetastatic osteosarcoma of the extremity wiyh pathologic fracture at presentation: local and systemic control by amputation or limb salvage after preoperative chemotherapy. Aceta orthop Scand 2003; 74: 449-54. 